\title{
The support needs of high-risk antenatal patients in prolonged hospitalisation
}

\section{Roxane Agnes Kent, MCur}

Labour Ward Unit Manager, MEDICLINIC KLOOF

Tel: +27 123674080

E-mail: roxanne.kent@mediclinic.co.za

\section{Mariatha Yazbek, PhD}

Lecturer. Department of Nursing, University of Pretoria, HW-Snyman, R8-13, Pretoria, South Africa

Tel: +27 825763558

Fax: +27123541490

E-mail: mariatha.yazbek@up.ac.za

\section{Tanya Heyns, PhD}

Senior Lecturer. Department of Nursing, University of Pretoria, HW-Snyman, R8-33, Pretoria, South Africa

Tel: +27 823873929

Fax: +27123541490

E-mail: Tanya.heyns@up.ac.za

\section{Isabel Coetzee, $\mathrm{PhD}$}

Senior Lecturer. Department of Nursing, University of Pretoria, HW-Snyman, R8-32, Pretoria, South Africa

Tel: +27711589045

Fax: +27 123541490

E-mail: Isabel.coetzee@up.ac.za

Word count of the text 4115

\section{Conflict of interest}

The authors have no conflicts of interest to disclose. 


\section{Acknowledgements}

The author is grateful to the patients who shared their needs during their prolonged hospital stay. The author would also like to thank $\operatorname{Dr} A E$ van der Wath for assisting with the coding of the data. All patient/personal identifiers have been removed.

\section{Keywords}

Support needs

High-risk antenatal patients

Prolonged hospitalisation

\section{Abstract}

Objective: to identify and describe the support needs of high-risk antenatal patients hospitalised for more than five days.

Design: a qualitative, explorative and descriptive design. In-depth interviews were conducted with pregnant women during their stay in hospital until data saturation was reached.

Setting: an antenatal unit in a private hospital in Gauteng Province, South Africa.

Participants: eleven antepartum women who had been hospitalised for five days or more and were of any gestation period.

Findings: three main themes emerged: (a) a need for social support; (b) improvement of the environment; and (c) assistance with emotional adaptation and acceptance of prolonged hospitalisation.

Key conclusion: prolonged hospitalisation of high-risk antenatal patients disrupts the usual adaptation to pregnancy. These patients develop specific needs during hospitalisation. Findings suggest that the length of hospitalisation influences the specific support needs of antenatal patients.

Implications for practice: this study identified a link between social and environmental support, emotional adaptation, acceptance of hospitalisation of highrisk antenatal patients and improvement of their health status. Through reflection on these themes, recommendations can be made and strategies implemented to meet the support needs of high-risk antenatal patients. 


\section{Introduction}

Most women anticipate a healthy pregnancy. Complications such as preeclampsia and premature labour may limit a woman from reaching term pregnancy and place her in a 'high-risk' category. Bed rest is a very common obstetrical management to restrict maternal activity even though there is minimal evidence that it decreases the risk of preterm delivery (Maloni, 2010). In addition Bigelow and Stone (2011) reviewed existing studies and found that there are no complications of pregnancy for which bed rest is consistently shown to be a beneficial intervention. A lack of objective research and randomised controlled trials analysing bed rest for a variety of complications of pregnancy is a major reason why up to $95 \%$ of obstetricians prescribe bed rest in their daily practice (Sciscione, 2010). Women with high-risk pregnancies are usually hospitalised for a prolonged period of time to ensure effective management of their condition and monitoring of the foetal well-being and growth.

After overcoming the initial shock, anxiety and disbelief of facing prolonged bed rest in hospital, the woman is faced with days or sometimes weeks of hospital confinement. Prolonged bed rest in hospital affects the antenatal patient and the family. These effects may include depression, loneliness, and separation from their families, which cause feelings related to a lack of social and emotional support during their pregnancies. The hospital environment has also been shown to lead to depression (Thakur and Blazer, 2008). Many women contribute a substantial portion of the family income and multiple studies have shown that prolonged hospitalisation puts the majority of families under some degree of financial strain (Bigelow and Stone, 2011).

A high-risk pregnancy can be stressful for the hospitalised pregnant woman, as the usual adaptation to her pregnancy is disrupted. She now has to cope with altered developmental tasks that have been created by the stress of her high-risk pregnancy (Heaman, 1998). Through the researcher's prolonged engagement with patients who had been hospitalised for a prolonged period, it became evident that all of them had support needs or developed specific support needs during their prolonged hospital stay.

The question arose: 'What are the support needs of high-risk antenatal patients on bed rest during prolonged hospitalisation?' The purpose of this study, therefore, was 
to explore and describe the specific needs of antenatal patients who had a prolonged hospitalisation as a result of pregnancy complications. Understanding of the specific support needs of these patients will enable the relevant healthcare professionals of the multi-disciplinary team to be aware of the specific support needs of high-risk antenatal patients and support them throughout their journey of prolonged hospitalisation.

\section{Methods}

A qualitative, explorative and descriptive design was used to conduct the study in a private hospital in Gauteng, a province in South Africa. Ethical approval was granted by the Ethics Committees of the Faculty of Health Sciences, University of Pretoria and a private hospital (S135/2010).

The qualitative researcher strives for an understanding of the whole, putting together a complex array of data. Qualitative studies produce findings closer to the data. Descriptive qualitative studies present comprehensive summaries of a phenomena or event. The researcher remains responsible to analyse and interpret the data as new insights can help shape application of the evidence generated to improve practice (Polit and Beck, 2012).

The main objective of this study was to explore the support needs of high-risk antenatal patients when hospitalised for a prolonged period of time to describe the specific needs of the patient as little is known about the topic. The patterns that emerged during data collection suggested similarities and divergences in the support needs of high-risk antenatal patients during prolonged hospitalisation. By understanding and interpreting the data, recommendations could be made at each of the themes that emerged to support patients hospitalised for a prolonged period.

\section{Population and sample}

Purposive sampling was used for this study. The rationale behind qualitative research is to purposefully select participants from sites that will best help the researcher understand the problem and the research question (Polit and Beck, 2012). 
Patients who had been hospitalised because of a high-risk pregnancy for five days or more and were 18 years and older and fluent in either Afrikaans or English were purposively recruited by the researcher. These patients gave valuable inputs about the specific supportive needs of antenatal patients hospitalised for more than five days. Written consent was obtained from the patients. The overseeing obstetricians were informed about the study.

\section{Data collection}

The labour unit admitted 348 women in a period of three months. Nineteen of the women (approximately 5\%) were hospitalised for more than five days. The women were approached five days after admission and were informed about the research. An information leaflet was given to them to read in their own time. The participants were again approached the following day and their questions were answered. The patients participated voluntarily and were informed of their right to confidentiality and to withdraw from the study at any time. All participants who denied permission were thanked for their time and were not approached again.

Eleven high-risk antenatal patients on bed rest five days and longer were interviewed. The in-depth interviews lasted between 25 and 40 minutes. Data saturation was reached after six interviews in respect of all the themes that emerged. An additional five interviews were conducted to confirm data saturation.

Consensus was reached between the researcher and the participants on a convenient time to conduct the face-to-face interviews. Face-to-face interviewing was the preferred data collection technique as it provided an opportunity to establish rapport with the interviewee and exchange information that could be difficult through methods such as surveys or questionnaires (Creswell, 2007). An interview guide with open-ended questions was designed to set the agenda and provide structure. Probing questions were used to clarify information when sufficient detail lacked. The interviews were conducted in one of the unoccupied private rooms in the antenatal ward as it provided a quiet and comfortable environment. Comfort of the antenatal patient was a priority to ensure that the hospitalised antenatal patient was at ease.

As illustrated in Table 1, most of the conditions the women were hospitalised for are life threatening to the mother and baby. The patients had all been placed on bed rest for monitoring of the mother and the baby, as well as to manage the condition. 
Bigelow and Stone (2011) describe a variety of physical and psychological adverse effects such as higher levels of combined anxiety, depression, hostility, profound boredom, depression, sensory disturbances and fatigue that women placed on bed rest have. It is therefore important to not only manage the high-risk antenatal condition but also to determine the specific physical and psychological needs of patients on bed rest for a prolonged period in hospital.

\section{Table 1}

Characteristics of participants

\begin{tabular}{|c|c|c|c|c|}
\hline & Age & Admission diagnosis & Gestational age & Type of pregnancy \\
\hline 1 & 22 & Antepartum Haemorrhage & 30 weeks & Singleton \\
\hline 2 & 30 & Antepartum Haemorrhage & 34 weeks & Singleton \\
\hline 3 & 25 & Antepartum Haemorrhage & 22 weeks & Singleton \\
\hline 4 & 24 & Preeclampsia & 20 weeks & Twin \\
\hline 5 & 26 & Preeclampsia & 22 weeks & Singleton \\
\hline 6 & 29 & Preeclampsia & 24 weeks & Singleton \\
\hline 7 & 32 & Preeclampsia & 26 weeks & Singleton \\
\hline 8 & 35 & $\begin{array}{l}\text { Preterm Premature Rupture of } \\
\text { Membranes }\end{array}$ & 28 weeks & Singleton \\
\hline 9 & 33 & $\begin{array}{l}\text { Preterm Premature Rupture of } \\
\text { Membranes }\end{array}$ & 25 weeks & Triplets \\
\hline 10 & 27 & $\begin{array}{l}\text { Preterm Premature Rupture of } \\
\text { Membranes }\end{array}$ & 28 weeks & Singleton \\
\hline 11 & 38 & Twin-to-twin transfusion & 31 weeks & Twin \\
\hline
\end{tabular}

Data analysis

The qualitative data collected during the in-depth interview was analysed utilising thematic content analysis (Braun and Clarke, 2006). The six steps provided by Creswell (2007) were utilised during data analysis. All the interviews were recorded and transcribed verbatim with the permission of the participants, as suggested by Polit and Beck (2012).

The data analysis was conducted independently by the researcher and a co-coder in order that interpreter bias was minimised. The analysis process involved reading and re-reading the transcripts for familiarisation by the researcher and co-coder with the content, for identification of code commonalities and differences, and for collating 
themes. Three main themes with subthemes emerged, which were then compared by the researcher and co-coder. Consensus was reached on the three main themes and subthemes that emerged from the data analysis.

\section{Trustworthiness}

Several strategies were used to increase the credibility of the results. Prolonged engagement established mutual trust between the researcher and the participants and an in-depth understanding of the phenomena under study. Similar experiences to those in the literature were reported by patients during prolonged hospitalisation. The findings could therefore be generalised. To improve reflexivity and not influence the findings, the researcher remained in contact with her own emotions, perceptions and background and reflected on her behaviour after every interview. She also adjusted her behaviour where necessary to not mislead the participants (Polit \& Beck, 2012). The interviews were audio-recorded and field notes were kept during interviews to describe and interpret the data correctly.

\section{Findings}

The findings of this study could allow healthcare professionals to identify and understand the different support needs of high-risk antenatal patients on prolonged bed rest. Understanding the support needs of these patients will enable healthcare professionals of the multi-disciplinary team to support patients throughout their journey of being admitted for a prolonged period of time. The three main themes that emerged from the interviews were: a need for social support; improvement of the environment; and assistance with emotional adaptation and acceptance of prolonged hospitalisation.

\section{A need for social support}

The need for social support was predominant. The thought of separation from a loved one is daunting for most patients during pregnancy. Separation from family members caused feelings of loneliness, isolation and feeling socially disconnected. 
The patients experienced separation from their loved ones negatively. Being around others did not relieve the feeling of loneliness.

I am suffering, it is not easy to leave your family and friends and just be here all the time...I am alone, I am meant to be experiencing this pregnancy with my husband... I am depressed and nobody comes to me; it makes me feel worse, I feel lonely...I cry every night like a child when my husband says good bye and leaves me behind...I feel lonely especially at night in this single bed on my own. P7.

Hospitalisation for a long period of time is very stressful and results in a need for social support to help the patient cope. Companionship and support from family members were lacking and patients experienced inadequate intimate relations and companionship in their interpersonal relationships. Patients also turned to staff for social support:

I really believe I need people to support me; I need my family and I need the staff to get me through this...I know the nurses are busy, but it would be nice to have some of their attention. I can talk to my roommate, but sometimes she is quiet and I think she feels the same as me. The doctor only comes in for half a minute every day just to say hello, then off he goes...I live for visiting times. P7.

Staff did not adopt the customs and practices appropriate to the cultural preferences of patients. Such patients missed these customs and felt lonely because they could not share their experiences:

It is difficult for me because of my religion...I need more privacy than a normal patient, so that is tough for me sometimes... just have a different way of life. P8.

\section{Improvement of the environment}

The environment to which patients were exposed played an important role in how they experienced hospitalisation. Throughout the study patients felt the environment 
was restrictive and lacked privacy. Reflection of how the patients perceived their restricted environment revealed the following:

....at home, you can do whatever you want... I am on strict bed rest, so I cannot even go outside or go to the coffee shop. P1.

In hospital, patients are removed from their normal environment where they are comfortable and can attend to their own needs. The ward routines, such as taking of vital signs and handing out medication, clashed with their personal daily routine and also disrupted their sleeping patterns. A hospital ward is a very public area, as staff and visitors are moving in and out. The patients felt trapped and struggled to adapt.

Privacy is widely accepted as a human right. The need of every human being to protect personal matters and relationships from the public is governed by a persons' value system (Malcolm 2005). The quotations below expressed how the patients perceived privacy during their hospitalisation:

...don't want to talk to him in the room because what I talk about can be heard...even though the other patients do not want to listen, they can still hear what I talk about...there is only a curtain between me and the patient next to me. P7.

Pregnancy and childbirth are for some patients very private experiences. The patients identified a lack of physical (the environment), social and informational privacy. Physically, the hospital environment did not allow for undressing:

I have to remain covered; it makes it tough. P8.

The patients could not control social activities such as rest and sleep, and interaction. They also became tired of reading and watching movies:

I can't even sleep and I will be woken up by some routine...I have to wake up every four hours to have my blood pressure taken, so I do not get enough sleep...I am always wondering what I can do next. P5. 
The outcomes of examinations were overheard by roommates as there were sometimes only curtains between patients. Healthcare professionals had to be made attentive to toning down their voices when disclosing personal information:

I really don't mind the girl I am sharing the room with, but I am self-conscious all the time and feel I cannot relax...But sometimes I feel exposed, because it is my illness, it is my pregnancy and now even the kitchen lady can hear what the doctor is saying about me. $P 7$.

\section{Emotional adaptation and acceptance}

Hospital admission is an emotional experience for most patients. Patients need to process and eventually accept their altered situation. All the patients admitted they felt depressed. Depression stemmed from feelings of sadness, helplessness and hopelessness. When the patients were diagnosed as having high-risk pregnancies and needed prolonged hospitalisation, these negative emotions of sadness, helplessness and hopelessness (set out below) were experienced:

The doctor told me that this illness affects the baby, so I was very concerned for my baby...I am really sad. Why did this have to happen to me?...I cried, I cried and cried and cried...My focus is more on getting through the day lately than actually getting the baby. P7.

Certain patterns of sadness were distinguished. There were moments of utter despair and also moments of delight when the condition of the patient improved. The long periods of hospitalisation also resulted in patients experiencing the absence of a cherished person or persons. Sadness contributed to a negative outlook of some patients on their current situation.

Sometimes I sit and wonder, what are the reasons this has to happen? And this makes me sad...I am at ease with the condition, but I am sad and there is sadness because of the separation of the family. P8. 
Feelings of helplessness caused insecurity, anxiety, negative experiences and emotions. Patients felt they could not take care of their own needs and nothing could be done to solve their problems. A loss in personal control contributed to the feelings of helplessness:

I would like to know exactly what is going to happen, when is it going to happen and I want to be able to control the outcome...I do not have expectations for this pregnancy any more. P2.

Hopelessness was experienced by the patents after they had heard their pregnancies were diagnosed as high risk. The patients thought all was lost and had no hope for the pregnancy. They expressed feelings of passivity and withdrawal and felt anger and a sense of being trapped by their condition, as nothing they could do would change the pregnancy outcome. The patients felt depressed, as they could not get out of the states of helplessness and hopelessness.

I feel I am stuck in a hole and no one can hear me...I feel like everything has been taken away - my baby, my husband, my job; I am not allowed to go home. P7.

\section{Discussion}

The aim of this qualitative, explorative and descriptive enquiry was to identify the physical and psychological needs of high-risk antenatal patients to determine their specific support needs during prolonged hospitalisation. High-risk pregnancy conditions lead to prolonged hospitalisation, as true adherence to bed rest is better controlled in a hospital setting. During hospitalisation the patient is exposed to the hospital environment and the multi-disciplinary team. On the basis of the data obtained from the study, the three themes that emerged from the interviews provided an explanation of the needs of high-risk antenatal patients who are hospitalised for a prolonged period of time. 
The need for social support derives from separation from the family. Patients on bed rest experience feelings of loneliness. Separation from family results in a lack of companionship and support from family members. Patients experience inadequate intimate relations and companionship in their interpersonal relationships (Cornwell and Waite, 2009). Without family support patients feel unsafe and may misinterpret the intentions of others and experience them as threatening. Patients react to stressful situations in isolation and in a passive way rather than in an active manner where they would be likely to seek help and support from others (Cacioppo et al., 2009).

Patients without social support feel extremely lonely. Loneliness is described as a feeling that emerges when social relationships are thought to be deficient (Doane and Adam, 2010). High-risk antenatal patients feel lonely when they are hospitalised for a long time. Their connection with, or to, others has been weakened, as partners or close family members are not involved (Cacioppo and Hawkly, 2009). Cultural norms should also be taken into account. If the hospital environment does not adopt certain customs and practices appropriate to the cultural preferences of patients, patients may end up missing these customs and feel lonely because they may not have any one to share their experiences with (Cacioppo and Hawkley, 2009). To feel respected and cared for patients also need to being listened to (Mattila et al., 2010). Social support and contact is a basic human need and has a direct beneficial effect on health that decreases stress processes and increases coping skills during prolonged hospitalisation.

It is therefore recommended that healthcare professionals adopt more flexible or unrestricted visiting hours for the significant others, family and close friends of patients. Such visiting hours will enable patients to have the experience of having their family and friends around them and gives them time to receive their support. $A$ further recommendation is 'sleep overs' on weekends if the unit is quiet. During sleep overs the husband and children are given the opportunity to sleep over in the room with the patient, which allows the patient to bond with her family and give her comfort and security. 
Improvement of the environment

The need to improve the environment stems from patients being removed from their usual environment into an environment that is totally unfamiliar, strange and restrictive. Multiple-bedded hospital rooms may be a comfort for some and a discomfort for others (Williams et al., 2007). When comparing the hospital to the patient's home, Richter et al. (2007) found patients felt trapped when depending on others. All the patients agreed it was easier to maintain personal control and emotional comfort at home. Hospitalisation took away their independence.

Pregnancy and childbirth is often a very private experience for patients and families. Ward routines such as vital sign monitoring and handing out of medication can clash with daily personal routine activities and also disrupt sleeping patterns. The nursing unit is a very public area and nursing staff, doctors and other members of the multi-disciplinary team, visitors and guests entering the unit unexpectedly. Maintaining privacy allows for a feeling of personal control and an opportunity for self-evaluation. Nursing practitioners should put into practice their understanding that privacy is a reasonable expectation to each and every person (Malcolm, 2005).

Privacy is multi-dimensional (Leino-Kilpi et al., 2002). The physical dimension (environment) should have areas for gynaecological examinations. Gynaecological examinations and undressing are uncomfortable, unpleasant and patients feel exposed (Leino-Kilpi et al., 2002). Curtains provide only visual barriers between patients in semi-private or bigger rooms but they do not serve as an auditory barrier (Yea-Pyng and Yun-Fang, 2010). The patients in the study emphasised that examination outcomes should not be overheard by roommates. The rooms were also not private enough during visiting hours. The social dimension is the autonomy of patients to control social interaction, rest and sleep. Martin (2005) points out that it is possible to predict the mood of the patient by assessing the way in which the curtains are drawn around the bed. A fully opened curtain means the patient welcomes contact with anyone; a half-drawn curtain indicates that only hospital staff can approach the patient; and, lastly, a fully drawn curtain indicates the patient does not want to be disturbed by anyone. The informational dimension ensures that private information is kept confidential. Some patients request health professionals to tone down their voices to prevent other patients from overhearing private 
conversations. A private room would ensure private exchange of information (YeaPyng and Yun-Fang, 2010).

Privacy of patients can be respected by examining patients in private rooms and not in rooms where other patients are also being admitted. It is further recommended that healthcare professionals should provide a special socialisation space to offer the patient an opportunity to break from the unit and its routine and relax with her family and friends. In addition, the patient should be allowed to bring items from home such as bedding, pictures or a side lamp to make the hospital room more 'homely'.

\section{Emotional adaptation and acceptance}

Patients inevitably need to emotionally adapt and accept their hospitalisation. A long hospital stay can provoke sadness, helplessness and hopelessness in patients. This can eventually result in depression, which may hinder patients from bonding effectively with their newborn babies. Sadness can arise when a person is faced with a disappointing situation that involves some sort of misfortune or loss (Bunkers, 2010). Sadness is very clearly identified as part of grief and is the main symptom of depression (Mouchet-Mages and Boyle, 2008; Bunkers, 2010). Negative emotions such as sadness have been found to influence the decision-making ability of a person. When in a negative mood, thoughts, judgements and perceptions are usually distorted and greater negativity is experienced. It is therefore understandable that antenatal patients who are admitted for a prolonged period of time will experience sadness, as admission implies that there are complications with their pregnancies. The absence of a cherished person or persons increases the feelings of sadness.

Patients also experience feelings of helplessness. Feelings of helplessness and a loss of control affect birthing expectations negatively. Admission during pregnancy causes more stress and negative emotions (Jordan and Murphy, 2009). Lee et al. (2007) describe helplessness as a feeling that nothing can be done to solve the problem (Lee et al., 2007). Patients also experience helplessness when they can no longer take care of their own needs (Richter et al., 2007).

Hopelessness as a psychological response to a negative event is described as a cognitive realm that involves negative expectations concerned with oneself and the future (Yurdagul and Oltuluoga, 2012). Hopelessness has two types of responses: 
affective and cognitive. Patients with affective responses show signs of passivity, a decreased involvement in care plans, a lack of initiative, outbursts of anger, and less hope and faith. Patients with cognitive responses believe problems will never be resolved, circumstances will never change and that the future looks bleak (Gurvoka and Ziqkova, 2011). The news antenatal high-risk patients receive can devastate them, change their entire outlook on pregnancy, and change their expectation of a positive pregnancy outcome to one that is uncertain or negative, depending on the severity of the diagnosis. These diagnoses threaten and possibly shorten patients' expectation of carrying to full term. The condition of the neonate, when born, may also become stressful for these patients.

Depression can be regarded as a common complication in pregnancy. A patient with depression in pregnancy has an increased chance of post-partum depression. It was confirmed that lower levels of support increase depressive symptoms in pregnancy, increase chances of post-traumatic stress, poor attitudes and a struggle to adjust to the pregnancy (Blanchard et al., 2009).

It is recommended that healthcare professionals provide opportunities for group sessions where patients can meet, share their sadness and find solace in one another. These sessions will assist patients to improve their problem-solving- and coping techniques.

Another important recommendation is to organise meetings between newly admitted patients and previously admitted patients. The previously admitted patients could give hope to those patients who have just been admitted. The newly admitted patients would have the opportunity to ask questions and clarify aspects such as ward routine and examinations they feel uncertain and anxious about.

\section{Conclusion}

Patients with high-risk pregnancy complications are hospitalised and bed rest is prescribed for prolonged periods despite the lack of supportive evidence in the literature. During prolonged hospitalisation, the patients in the study developed social-, environmental-, emotional- and adaptation needs and found it difficult to accept prolonged hospitalisation. The patients demonstrated different support needs 
depending on the duration of their hospital stay and cultural background. The support needs also differed from one patient to the next.

In conclusion, the support needs that emerged from the high-risk patients on bed rest of a hospital in the private sector emphasised that humans are fundamentally social creatures and need others (especially family members) to survive. Feelings such as loneliness are the result of a shortfall in one's social resources and the disruption of companionship and support structures. If these support needs are recognised, recommendations can be made to address them and implement them in the units. Implementing these recommendations will enable antenatal patients to adapt to and cope better with prolonged hospitalisation.

\section{References}

Bigelow, C., Stone, J., 2011. Bed rest in pregnancy. Mount Sinai Journal of Medicine 78, 291-302.

Blanchard, A., Hodgson, J., Gunn, W., White, E.J., White, M., 2009. Understanding Social Support and the Couple's Relationship Among Women with Depressive Symptoms in Pregnancy. Issues in Mental Health Nursing 30, 764-776.

Braun, V., Clarke, V., 2006. Using thematic analysis in psychology. Qualitative Research in Psychology 2, 77-101.

Bunkers, S., 2010. The lived experience of feeling sad. Nursing Science Quarterly 23 (3), 231-239.

Caccioppo, J.T., Hawkley, L.C., 2009, Perceived social isolation and cognition. Trends in Cognitive Science 13, 447-454.

Caccioppo, J.T., Norris, C.J., Decety, J., Monteleone, G., Nusbaum, H., 2009. In the eye of the beholder: individual difference perceives social isolation predict regional brain activation to social stimuli. Journal of Cognitive Neuroscience 1, 83-92.

Cornwell, E.Y., Waite, L.J., 2009. Social disconnectedness, perceived isolation, and health among older adults. Journal of Health and Social Behaviour 50, 31-48.

Creswell, J.W., 2007. Qualitative Inquiry and Research Design: Choosing Among Five Approaches. 2nd edn. Sage Publications, London. 
Doane, L.D., Adam, E.K., 2010. Loneliness and cortisol: momentary, day-to-day, and trait Associations. National Institute of Health, Journal of Health and Social Behaviour 3, 430-441.

Gurkova, E., Ziakova, K., Cap, J., 2011. Content validation of hopelessness in Slovakia and Czech Republic. International Journal of Nursing Terminologies and Classifications 22, 33-39.

Heaman, M., 1998. Psychosocial impact of high-risk pregnancy: hospital and home care. Clinical Obstetrics and Gynecology 41 (3), 626-639.

Jordan, R.G., Murphy, P.A., 2009. Risk assessment and risk distortion: finding the balance. Journal of Midwifery and Women's Health.54, 191-200.

Lee, C.Y., Low, L.P.L., Twinn, S., 2005. Older men's experiences of sleep in the hospital. Journal of Clinical Nursing 16, 336-343.

Leino-Kipli, H., 2002. Maintaining privacy on post-natal wards: a study in five European counrties. Journal of Advanced Nursing 37, 145-154.

Malcolm, H. A., 2005. Does privacy matter? Former patients discuss their perceptions of privacy in shared hospital rooms. Nursing Ethics 12, 156- 166.

Maloni, J. A., 2010. Antepartum bed rest for pregnancy complications: Efficacy and safety for preventing preterm birth. Biological Research for Nursing 12(2), 106124.

Martin, J., 2005. Notes on the tension between privacy and surveillance in nursing. Online Journal of Issues in Nursing 10 (2), 110-130.

Mattila, E., Kaunonen, M., Ollikainen, J., Astedt-Kurki, P., 2010. Support for hospital patients and associated factors. Scandinavian Journal of Caring Sciences 24, 734-745.

Mochet-Mages, S., Bayle, F.J., 2008. Sadness as an integral part of depression. Dialogues in Clinical Neuroscience 10, 321-326.

Polit, D.F., Beck, C.T., 2012. Nursing research: generating and assessing evidence for nursing practice. 9th ed. Williams \& Wilkins, Philadelphia.

Richter, M.S., Parkes, C., Chaw-Kant, J., 2007. Listening to the voices of hospitalized high-risk antepartum patients. Journal of Obstetric, Gynecologic and Neonatal Nursing 36 (4), 313-318.

Sciscione, A. C., 2010. Maternal activity restriction and the prevention of preterm birth. American Journal of Obstetrics and Gynecology 202, 23 e1-e5. 
Thakur, M., Blazer, D.G., 2008. Depression in long-term care. Journal of American Medical Directors Association 9, 82-87.

Williams, A.M., Dawson, K., Kristjanson, L.J., 2007. Exploring the relationship between personal control and the hospital environment. Journal of Nursing Care 17, 1601-1609.

Yea-Pyng, L., Yun-Fang, T., 2010. Maintaining patients' dignity during clinical care: a qualitative interview study. Journal of Advanced Nursing. 67 (2), 340-348.

Yurdagul, Y., Oltuluoglu, H., 2012. Social support and hopelessness in women undergoing infertility treatment in Eastern Turkey. Public Health Nursing. 29, 99104. 